\title{
AN INTRODUCTION TO SOME NEW RESULTS IN BIOINFORMATICS AND COMPUTATIONAL BIOLOGY
}

\author{
LIMSOON WONG \\ Managing Editor \\ Published 17 April 2013
}

DNA markers are frequently used to analyze crop varieties. In this issue, Fujii et al. $(2013)^{1}$ describes an algorithm and its associated software, MinimalMarker, for producing a minimal set of DNA markers for characterizing a given set of crops. The program can be used with both dominant and co-dominant markers regardless of the number of alleles, including short sequence repeats.

Discovering the linkage and association of a gene to a disease has been an active area of research. ${ }^{2}$ One challenge here is the identification of disease alleles based on SNP genotypes, which often requires a large number of samples. As the identification of disease alleles based on haplotype data is much easier, one route to the challenge is to first infer haplotypes from SNP genotypes and other information. ${ }^{3-5}$ In this issue, Sabaa et al. $(2013)^{6}$ presents iBDD, a program for determining all distinct haplotype allele identity-by-descent sharings in a single scan of the genome, even for very complex pedigree genotype datasets. In another article in this issue, Abad-Grau et al. $(2013)^{7}$ show how to use haplotype similarity as prior knowledge to group and classify haplotypes into high- or low-risk ones effectively. Another interesting challenge here is the discovery of more candidate genes associated to a disease given a few known "seed" genes. ${ }^{8}$ In this issue, Hossain et al. $(2013)^{9}$ present a procedure to tackle this challenge by identifying genes that are correlated with a seed gene in microarray experiments.

The growing number of fully sequenced genomes has enabled many comparative genomics studies. One challenge here is the inference and reconciliation of past events - e.g., gene duplications, losses, and transfers — in the species evolutionary history. An exponential number of scenarios are possible and have to be considered. In this issue, Scornavacca et al. $(2013)^{10}$ introduce a reconciliation graph to compactly represent these possible scenarios and show that this representation has polynomial size. They also provide an algorithm to find reconciliation graphs in polynomial time. 
Intrinsically disordered proteins can interact with many targets and thus fulfil important roles in numerous cellular processes. While methods for predicting disordered residues are generally successful, they often do not have consistent performance on long disordered regions. ${ }^{11}$ In this issue, Deiana \& Giansanti $(2013)^{12}$ show that the inconsistent performance is due to false positives; and they devise a simple procedure to improve precision by determining the disagreement among several popular disorder predictors and re-tuning their discriminative thresholds.

The RNA inverse folding problem is to determine an RNA sequence that folds into a given target RNA secondary structure. While there has been much work on predicting RNA structure given its sequence and detecting motifs in RNA structures, ${ }^{13-15}$ the RNA inverse folding problem has received less attention. In this issue, Garcia-Martin et al. $(2013)^{16}$ present a constraint programming approach to this problem. This is the first instance of a complete RNA inverse folding approach that supports the specification of a wide range of design constraints.

Imaging processing has a broad range of applications in cell biology. ${ }^{17}$ For example, it is a time-consuming task in cell biology to extract fluorescence intensity profiles of single cells from image data. In this issue, Herzog et al. $(2013)^{18}$ present a method for segmenting single rod-shaped cells from image data and extracting fluorescence intensity profiles from the cell under challenging conditions such as low spatial resolution and having no information on the imaging system.

Due to advances in high-throughput technologies, large amounts of proteinprotein interactions data have become available ${ }^{19}$ for a variety of applications such as identifying functional modules,${ }^{20}$ inferring drug targets, ${ }^{21}$ etc. In this issue, Srihari and Leong $(2013)^{22}$ present a comprehensive survey on an important application of protein interaction network: the discovery of protein complexes. The survey puts emphasis on the evolution of protein complex prediction methods toward the incorporation of more biological background knowledge and also highlights several interesting open problems in protein complex prediction that are still unaddressed by current methods. Much of the currently available protein interaction data and approaches are concerned with the interactions of proteins within a single organism or a single cell. There are significantly fewer studies on the interactions of proteins between two organisms, such as in host-pathogen interactions. In this issue, Zhuo and Wong $(2013)^{23}$ present a timely systematic survey on the development in computational studies of host-pathogen interactions. The survey offers a very comprehensive summary of prediction of host-pathogen protein-protein interactions and properties of host-pathogen interactions, as well as a nice review of database and software tools for host-pathogen interaction data collection, integration and analysis.

Systems biology is an emerging holistic approach to model and study the complex interactions within a biological system. ${ }^{24}$ In this issue, Kuhn and Gennemark $(2013)^{25}$ review mathematical models of yeast's response to osmotic stress. It is an excellent overview of the different modeling techniques. It offers an informative and insightful discussion on the requirements, advantages, and limitations of these techniques. 


\section{References}

1. Fujii H, Ogata T, Shimada T et al., Minimal marker: An algorithm and computer program for the identification of minimal sets of discriminating DNA markers for efficient variety identification, Journal of Bioinformatics and Computational Biology 11(2):1250022, 2013.

2. Limviphuvadh V, Chua LL, Eisenhaber F et al., Is LGI2 the candidate gene for partial epilepsy with pericentral spikes? Journal of Bioinformatics and Computational Biology 8(1):117-127, 2010.

3. Li J, Jiang T, A survey in haplotyping algorithms for tightly linked markers, Journal of Bioinformatics and Computational Biology 6(1):241-259, 2008.

4. Li X, Li J, An almost linear time algorithm for a general haplotype solution on tree pedigrees with no recombination and its extensions, Journal of Bioinformatics and Computational Biology 7(3):521-545, 2009.

5. Wang WB, Jiang T, Inferring haplotypes from genotypes on a pedigree with mutations, genotyping errors, and missing alleles, Journal of Bioinformatics and Computational Biology 9(2):339-365, 2011.

6. Sabaa H, Cai Z, Wang Y et al., Whole genome identity-by-descent determination, Journal of Bioinformatics and Computational Biology 11(2):1350002, 2013.

7. Abad-Grau MM, Medina-Medina N, Moral S et al., Increasing power by using haplotype similarity in a multimarker transmission/disequilibrium test, Journal of Bioinformatics and Computational Biology 11(2):1250014, 2013.

8. Ochagavia ME, Miranda J, Nazabal M et al., A methodology based on molecular interactions and pathways to find candidate genes associated to diseases: Its application to schizophrenia and Alzheimer's disease, Journal of Bioinformatics and Computational Biology 9(4):541-557, 2011.

9. Hossain A, Willan AR, Beyene J, A flexible nonparametric approach to find candidate genes associated with disease in microarray experiments, Journal of Bioinformatics and Computational Biology 11(2):1250021, 2013.

10. Scornavacca C, Paprotny W, Berry V, Ranwez V, Representing a set of reconciliations in a compact way, Journal of Bioinformatics and Computational Biology 11(2):1250025, 2013.

11. Peng K, Vucetic S, Radivojac P et al., Optimizing long intrinsic disorder predictors with protein evolutionary information, Journal of Bioinformatics and Computational Biology 3(1):35-60, 2005.

12. Deiana A, Giansanti A, Tuning the precision of predictors to reduce overestimation of protein disorder over large datasets, Journal of Bioinformatics and Computational Biology 11(2):1250023, 2013.

13. Wan XF, Lin G, Xu D, RNALL: An efficient algorithm for predicting RNA local secondary structural landscape in genomes, Journal of Bioinformatics and Computational Biology 4(5):1015-1032, 2006.

14. Sulc P, Wagner A, Martin OC, Quantifying slow evolutionary dynamics in RNA fitness landscapes, Journal of Bioinformatics and Computational Biology 8(6):1027-1040, 2010.

15. Hua L, Wang JTL, Ji X et al., A method for discovering common patterns from two RNA secondary structures and its application to structural repeat detection, Journal of Bioinformatics and Computational Biology 10(4):1250001, 2012.

16. Garcia-Martin JA, Clote P, Dotu I, RNAiFold: A constraint programming algorithm for RNA inverse folding and molecular design, Journal of Bioinformatics and Computational Biology 11(2):1350001, 2013. 
17. Ohtani M, Saka A, Sano F et al., Development of image processing program for yeast cell morphology, Journal of Bioinformatics and Computational Biology 1(4):695-710, 2004.

18. Herzog A, Voss B, Keilberg D et al., A strategy for identifying fluorescence intensity profiles of single rod-shaped cells, Journal of Bioinformatics and Computational Biology 11(2):1250024, 2013.

19. Ng SK, Tan SH, Discovering protein-protein interactions, Journal of Bioinformatics and Computational Biology 1(4):711-742, 2004.

20. Sohaee N, Forst CV, Identification of functional modules in a PPI network by bounded diameter clustering, Journal of Bioinformatics and Computational Biology 8(6):929-943, 2010 .

21. Ivanov AS, Gnedenko OV, Molnar AA et al., Protein-protein interactions as new targets for drug design: Virtual and experimental approaches, Journal of Bioinformatics and Computational Biology 5(2b):579-592, 2007.

22. Srihari S, Leong HW, A survey of computational methods for protein complex prediction from protein interaction networks, Journal of Bioinformatics and Computational Biology 11(2):1230002, 2013.

23. Zhou H, Jin J, Wong L, Progress in computational studies of host-pathogen interactions, Journal of Bioinformatics and Computational Biology 11(2):1230001, 2013.

24. Liu B, Thiagarajan PS, Modeling and analysis of biopathways dynamics, Journal of Bioinformatics and Computational Biology 10(4):1231001, 2012.

25. Kühn C, Gennemark P, Modelling yeast osmoadaptation at different levels of resolution, Journal of Bioinformatics and Computational Biology 11(2):1330001, 2013. 